\title{
Synthetic Aperture Radar for Lane Boundary Detection in Driver Assistance Systems
}

\author{
Daniel Clarke, Daniel Andre ${ }^{1}$ and Feihu Zhang ${ }^{2}$
}

\begin{abstract}
In this paper we investigate the feasibility for using a Synthetic Aperture Radar (SAR) to detect radar scatterers in support of advanced driver assistance systems. Specifically, we consider the detection of radar scatterers physically embedded into lane and carriageway boundaries similar to way optical retroreflectors (cats eyes) are used in present infrastructure. We use simulations to generate high resolution SAR images for detecting and localizing radar scatterers. The simulated results presented here highlight the feasibility of the technique and provide a platform for further investigation. This paper facilitates the realization of the role of modified infrastructure for improving the sensing capability of highly assisted and autonomous vehicles.
\end{abstract}

\section{INTRODUCTION}

Presently, there is significant interest into autonomous vehicles, both from a consumer and research perspective and many organizations ranging from universities through to automotive manufacturers are investing heavily in this technology. Much of this research is focused on developing the sensing technology for providing high fidelity situational awareness of the dynamic, cluttered and often obscured environment around a vehicle. One such area of interest is the detection of lane and carriageway boundaries for lane-departure warning systems. Most lane and carriageway detection systems utilize the following steps: detect and extract lane marking elements; produce a geometrical model estimation; and filter/track the parameters of the geometrical model along an image sequence [1].

The primary focus of this paper is on the first step of detecting and extracting the lane marking elements. We impose this limit because the subsequent steps of geometric modeling and tracking/filtering merely utilize the measurement data provided by the marker detection sensor. At present a majority of the research in this area uses cameras and computer vision techniques to extract the markings which are painted onto the road. However, these techniques are dependent upon the camera maintaining good visibility of the road markings, something which cannot always be guaranteed (e.g. in conditions of poor lighting, poor weather etc.). As autonomous vehicle functionality matures, it is likely that infrastructure will be developed specifically to accommodate driver-less vehicles. In this paper we propose a methodology for using vehicle-mounted Synthetic Aperture

\footnotetext{
${ }^{1}$ Daniel Clarke and Daniel Andre are with the The Centre for Electronic Warfare, Cranfield University, The Defence Academy of the United Kingdom, Shrivenham, Swindon, SN6 8EH, UK. d.s.clarke@cranfield.ac.uk and d.andreecranfield.ac.uk

${ }^{2}$ Feihu Zhang is with fortiss an Institute of the Technical University of Munich, Munich, zhang@fortiss.org
}

Radar (SAR) to detect radar scatterers physically embedded into the road similar to the way in which optical retroreflectors (i.e. cats-eyes) are currently used.

\section{Generalized Description of THE Problem}

\section{A. Current Lane Marker Detection Techniques}

Modern infrastructure has been developed for use by human drivers and as such, many modern ADAS systems rely on computer vision technologies for most of their operation. This is especially prevalent in systems which detect and extract lane and carriageway boundaries, which are bands of paint with particular lengths and widths painted onto the road. This field of research is well established and there are a number of techniques which consider both the photometric and geometric characteristics of the markers. A dedicated review of computer vision techniques for lane marking extraction is given in [2]. Some specific techniques are listed herein in order to motivate the problem statement of this paper. Adaptive thresholding is used in [3] and [4], where each pixel within an image is tested against a threshold value (which is dynamic over the image) in order to segment specific features. The adaptive nature of the thresholding means that areas resulting from strong illumination gradients (e.g. shadows or saturation) are similarly segmented. Steerable filters use a convolution kernel rotated to different angles under adaptive control to calculate the filter response at different orientations, and are used in [5] and [6] for extracting lane features. Finally, color segmentation (as used in [7]) partitions the image into super-pixels to effectively locate the object boundaries from which lane markers are extracted.

However, each of the above techniques described above relies solely on the use of cameras for their operation for specific detection and extraction of the lane markers. Unfortunately, automotive grade camera systems perform poorly in conditions of poor visibility (e.g. low light, poor weather etc.). Although several commercial products have already emerged in the market, it remains challenge to fully detect lanes in all scenarios [8].

\section{B. Automotive Radar Technology}

The use of radars in vehicles is well established and they form a principal element in both safety and driver assistance functions, mainly due to their high range resolution and all weather capability. Most existing automotive radar systems (e.g. [9], [10], [11]) operate a Frequency Modulated Continuous Wave signal in the $\mathrm{mm}$ band (usually 
around either $24 \mathrm{GHz}$ or $75-80 \mathrm{GHz}$ ), operating in a multistatic mode. This mode of operation enables them to operate with good performance as multi-range detectors, but only provides a coarse azimuthal resolution [12]. For highly assisted and autonomous vehicles, it is perceived that current serial production automotive radars are unable to provide the required image like capabilities [13].

The use of SAR in the automotive domain is currently rather limited. This is principally due to the fact that the environment is highly cluttered and that many targets of interest are generally poor radar scatterers. Therefore, there remain very few examples of automotive SAR. One such automotive SAR is presented in [14], where the authors use an ultrawide band, low Frequency SAR on an unmanned vehicle to detect man made concealed obstacles (e.g. minefields, booby traps, ditches and bodies of water). While this technology is useful for military surveillance applications, it does not offer sufficient performance for the application envisaged herein. In [15], the authors present an vehicle mounted, side looking $\mathrm{X}$-band SAR, however the longer wavelength mean that its use is principally limited to high resolution image formation for land observation. In [16] the authors propose a novel technique for compensating the image formation for the vehicle motion, but do not propose any specific autonomous functionality.

At present, many highly assisted and autonomous driving applications rely on cameras and computer vision algorithms, as present infrastructure has been developed around human drivers who rely on biological vision. Due to limited budgets and industrial economics, most research focuses on driving current vehicles in current infrastructure. However there do exist projects, which are considering the role infrastructure could play in autonomous vehicles, both from the fundamental academic principles and practicable engineering considerations. This work ranges from future infrastructure projects such as the Cranfield University intelligent highways [17], through to works which seek to utilize sensors in the infrastructure for cooperative situational awareness (e.g. [18], [19], [20]).

\section{Radar Reflectors}

The aim of this paper is to highlight that SAR can be used to detect highly reflective scatterers embedded into the infrastructure, acting as a remotely sensed track along which an autonomous vehicle can navigate. Our scenario envisages that radar reflectors are physically built into either kerbstones lining the sides of the roads, or cats-eye like devices which are embedded into the road surface at lane/carriageway boundaries. The physical form of the radar scatterer should be something that reflects the maximum energy back towards the radar transceiver, thus overcoming one of the main challenges of automotive SAR. There are many types of scatterers which may be of interest to this project, including retro-reflective trihedrals or simple ball bearings. Typically the scatterers will be of a size which is proportional to the wavelength (where $75 \mathrm{GHz}$ is a wavelength of approximately
$4 \mathrm{~mm}$ ) and thus can be realistically used within the infrastructure.

On the vehicle, the use of both a forward looking and side looking radar system is used to detect the scatterers, build a model of their layout and subsequently locate the vehicle in reference to those markers. Fig. 1, indicates the proposed layout of radar sensors on the vehicle and scatterers/reflectors embedded into the lane and carriageway boundaries. The configuration of the radar sensors is described in section IV.

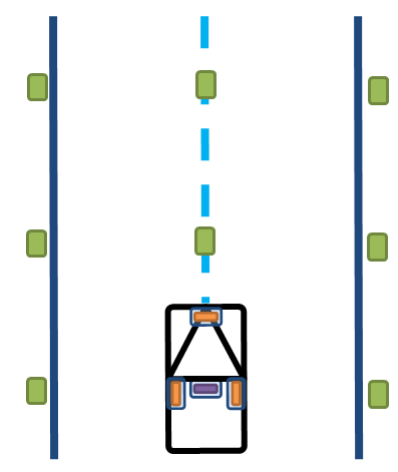

Fig. 1: The proposed configuration. The green squares are the trihedral markers, while the orange boxes are the SAR sensors. This figure is not presented to scale and the different configurations tested are given in section IV.

Within this paper, we consider only the scenario and configuration which would allow the formation of an SAR image for the detection of the markers. The model fitting and pose tracking are not considered as part of this paper. The forward looking radar is expected to detect markers at ranges of $<200 \mathrm{~m}$ while the side looking radar system will detect markers at ranges of $<2 \mathrm{~m}$.

\section{PROPOSED RADAR CONFIGURATION}

\section{A. Synthetic Aperture Radar Principles}

Synthetic aperture radar is a technique which has been used in the remote sensing domain for a number of decades. The operating principles of traditional monostatic radar provide a range profiles with excellent accuracy and resolution. However, the azimuthal precision is typically very coarse, relying on the gain pattern of the receive antenna. Synthetic aperture radars utilize the motion path of the host platform to electronically simulate a large aperture and provide a much higher cross-range resolution. Thus, SARs are able to generate image quality range and cross-range profiles. In this section, we describe the fundamental steps for building such a profile, following the terminology given in [21].

For a SAR sensor following a motion path, observing a stationary target, the antenna phase center is given as

$$
r_{a}(\tau)=\left[x_{a}(\tau), y_{a}(\tau), z_{a}(\tau)\right]^{T}
$$

with $\tau$ denoting the synthetic aperture. The coordinate framework is defined in reference to a scene origin corresponding to the SAR motion compensation point whose distance to the phase center is given as 


$$
d_{a}(\tau)=\sqrt{x_{a}^{2}(\tau)+y_{a}^{2}(\tau)+z_{a}^{2}(\tau)}
$$

A stationary target with position $r=\left[x_{a}, x, y, z\right]^{T}$, has a distance to the phase center given as

$$
d_{a_{0}}(\tau)=\sqrt{\left(x_{a}(\tau)-x\right)^{2}+\left(y_{a}(\tau)-y\right)^{2}+\left(z_{a}(\tau)-z\right)^{2}}
$$

The radar periodically transmits pulses which are reflected by all scatterers in the scene, with some of the energy received by the radar. Within a synthetic aperture of $N_{p}$ positions, pulses are transmitted in the sequence $\tau_{n}$ where $n=\left(1,2, \ldots, N_{p}\right)$ and used to form the image. The output of the receiver at time $\tau_{n}$ is is a sequence of frequency samples delayed by the round-trip time to the target. There are $K$ frequency samples per position spread over the signal bandwidth, with the frequency values represented by $f_{k}$ where $k=(1,2, \ldots, K)$. Thus, the receiver output from the target located at $\mathrm{r}$, with a received amplitude $A\left(f_{k}, k\right)$ can be derived from the wave equation and given as

$$
s\left(f_{k}, \tau_{n}\right)=A\left(f_{k}, \tau_{n}\right) \exp \left(\frac{-i 4 \pi f_{k} \Delta R\left(\tau_{n}\right)}{c}\right)
$$

From equation 4 , the matched filter can be built. If we assume a single point source scatterer (i.e. a constant amplitude $A\left(f_{k}, \tau_{n}\right)=A_{0}$, the matched filter response $I(r)$ at location $r$ is given by

$$
I(r)=\frac{1}{N_{p} K} \sum_{n=1}^{N_{p}} \sum_{k=1}^{K} s\left(f_{k}, \tau_{n}\right) \exp \left(\frac{-i 4 \pi f_{k} \Delta R\left(\tau_{n}\right)}{c}\right)
$$

To form an image, equation 5 is computed for each pixel location. However, this requires the differential range $\Delta R\left(\tau_{n}\right)$ to be computed for each pixel and for each pulse and results in a complexity of $\mathcal{O}\left(N^{4}\right)$ for a 2D image. There exist a number of techniques for implementing this which vary in both complexity and computational expense, [22] provides a full description and comparison. The Back projection algorithm forms the basis for the simulations presented in the remainder of this paper, however their full derivation is out of scope for this paper and can be found in [21].

As an example of the image generate by the SAR is given in Fig. 2. This image was simulated using a baseline of 16 SAR receiver locations equally spaced along a straight line $13 \mathrm{~cm}$ apart, observing a single isotropic scatterer located at the origin of the scene. This image was formed with the parameters $N_{p}=16$ and $K=2^{11}$. The radar system was simulated with a center frequency of $24 \mathrm{GHz}$, bandwidth of $150 \mathrm{MHz}$. The parameters provide a range resolution of $1 \mathrm{~m}$, and a cross range resolution of $0.28 \mathrm{~m}$. The color-scale in the image is essentially providing a measure of the brightness (or radar cross section) of the scatterers within the screen.

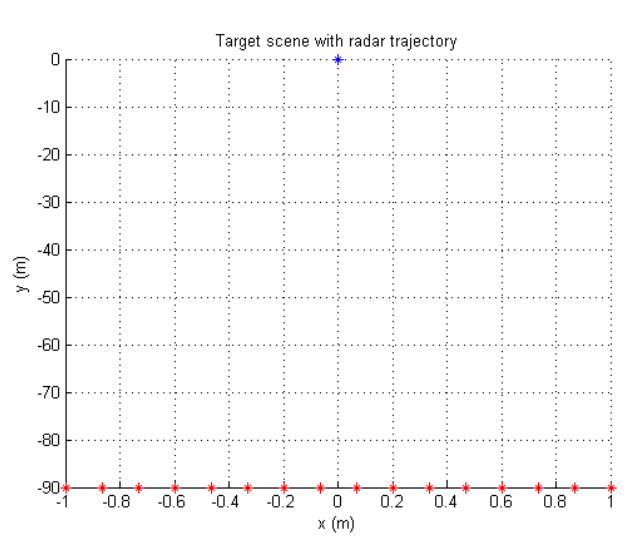

(a) Simulation Set Up

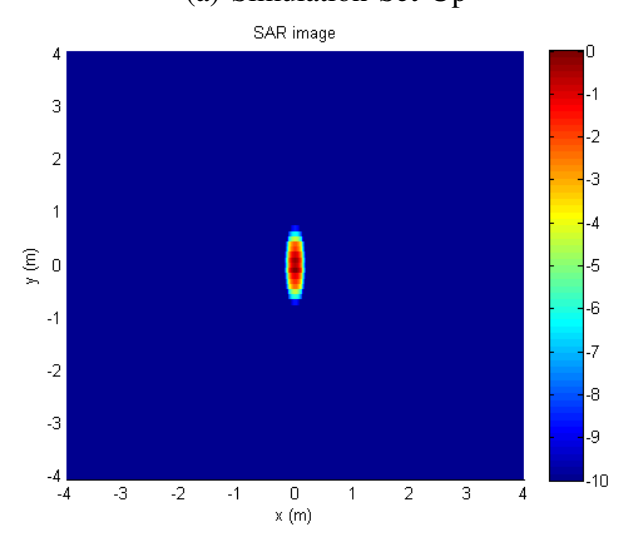

(b) Simulation Results

Fig. 2: This image simulates a SAR baseline observing a single isotropic scatterer. The target scene is shown on the top, where the radar receiver locations are given by the red star, the target is given by the blue star. The SAR image shown on the bottom indicates the relative signal strength in $\mathrm{dB}$ over a limited portion of the scene space $(8 \mathrm{x} 8 \mathrm{~m}$ centered at the origin).

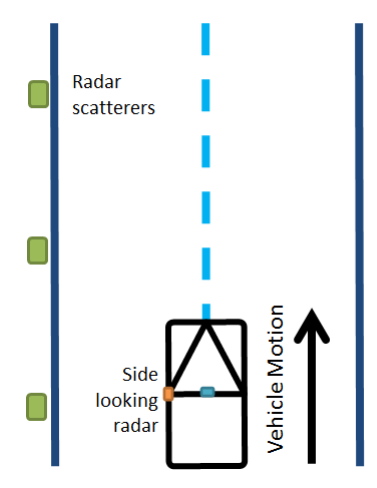

Fig. 3: The proposed configuration. The green squares are the radar reflectors, while the orange boxes are the SAR sensors. This figure is not presented to scale and the different configurations tested are given in section IV.

\section{Simulation Results}

In this section, we use the theoretical framework developed in section III to simulate SAR images. The simulated SAR 
images are used as a preliminary study to prove the feasibility of an automotive SAR with physical reflectors can be used for lane and carriageway boundary detection. Within these scenarios, we assume perfect isotropic reflectors and do not insert clutter, noise or false targets into the scene. Furthermore, we do not consider the techniques which would model and track the location of the markers within the SAR images.

\section{A. Side Looking Radar}

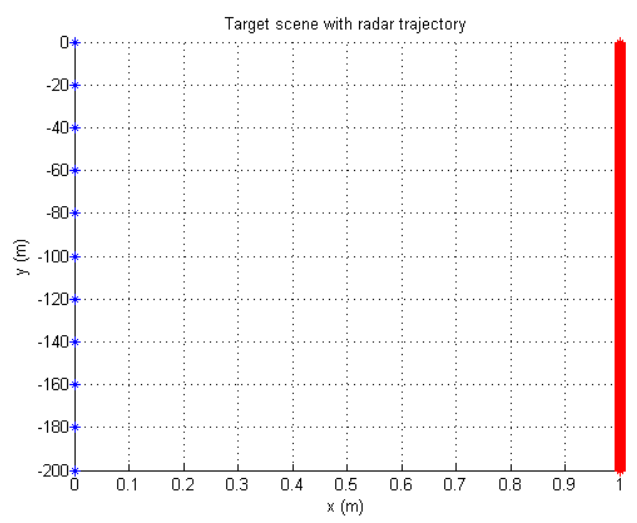

Fig. 4: The scene set up for the side looking SAR example. The locations at which pulses are recorded is given by the red stars, while the radar reflectors are given by the blue stars.

The first scenario that we investigate is ability of side looking SAR to be able to detect and form an image of scatterers to the side of the vehicle. This scenario considers a vehicle driving along a straight line, with a single side looking radar sensor used to generate the SAR image and is shown in Fig. 3. The SAR is configured with a center frequency of $24 \mathrm{GHz}$, bandwidth of $150 \mathrm{MHz}$ (typical of a modern automotive radar) and ability to measure 64 locations per second. The markers are placed at the nominal lane boundary (which is again modeled as a straight line) at $20 \mathrm{~m}$ intervals.

Fig. 4 indicates the scene set up, while Fig. 5 shows the SAR image for a vehicle traveling along a straight road for $200 \mathrm{~m}$ at velocities of $10 \mathrm{~m} / \mathrm{s}(36 \mathrm{kph}) 20 \mathrm{~m} / \mathrm{s}(72 \mathrm{kph})$ and 40 $\mathrm{m} / \mathrm{s}(144 \mathrm{kph})$. This results in a number of positions $N_{p}=$ $(640,320,160)$ respectively for the SAR image formation. All scenarios utilized a number of frequencies $K=2^{12}$.

We can see that within the side looking scenario, there is sufficient resolution both in the range and cross-range to adequately detect the markers using a variety of image processing algorithms. While the markers do appear to have a stretched, range profile, the peaks remain distinct. Furthermore, Fig. 5 show that as the simulated vehicle speed increases, the number of positions $N_{p}$ used to form the SAR image decreases and so does the resolution. While the results presented here are not comprehensive, they are sufficient to indicate the feasibility of a moving vehicle forming a SAR image to detect lane and carriageway markers to the side of the vehicle.

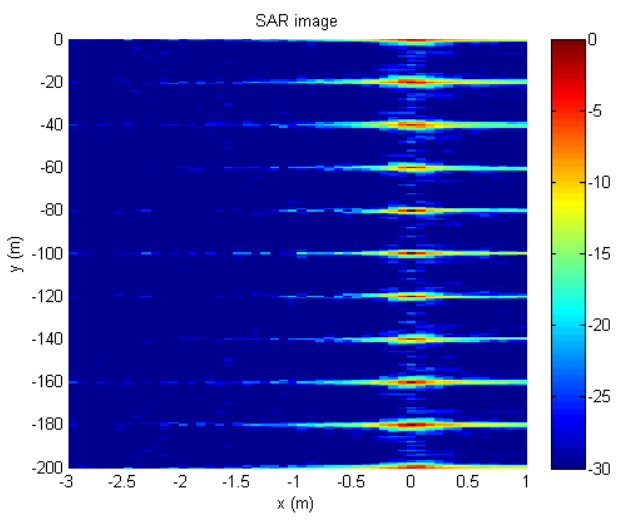

(a) SAR image for $10 \mathrm{~ms}^{-1}$

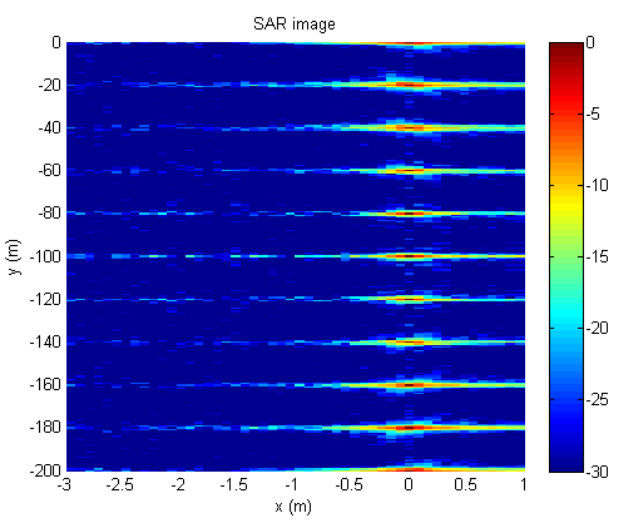

(b) SAR image for $20 \mathrm{~ms}^{-1}$

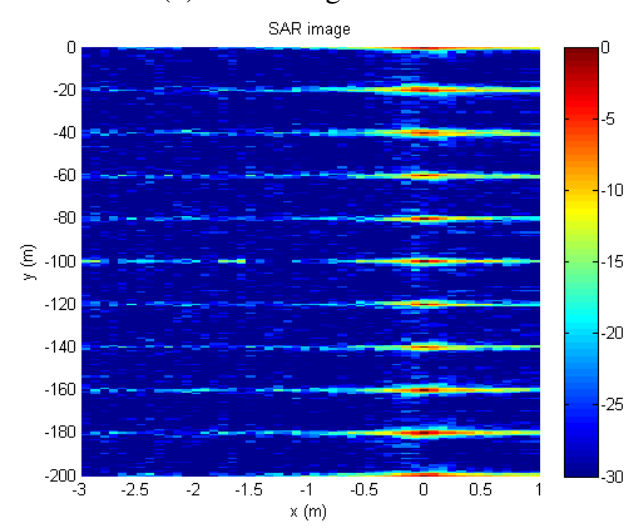

(c) SAR image for $40 \mathrm{~ms}^{-1}$

Fig. 5: A simulated scenario featuring a vehicle with a SAR observing lane markers to the side of the vehicle. As the vehicle travels faster, fewer pulses $\left(N_{p}\right)$ are used to form the image.

Further investigation has shown values of $N_{p}<100$ generally result in a SAR image that is too noisy for useful analysis. This is essentially because the cross range resolution is angular such that $\Delta \leq \theta \frac{c}{2 L_{c r} F_{c}}$, where $L_{c r}$ is the cross range resolution and $F_{c}$ is the center frequency. As such there is a minimum number of positions at which measurements 
should be taken. Future work should investigate this further in order to inform the design principles of the radar.

\section{B. Forward looking radar}

The second scenario that we wish to investigate is the ability of a forward looking SAR to be able to detect multiple rows of scatterers in front of the vehicle. This scenario considers a vehicle driving along a straight line, with multiple radar receiver elements placed uniformly across the front of a vehicle. The scene for this model is illustrated in figure 6. Throughout this section, we utilize a radar with a center frequency of $24 \mathrm{GHz}$, a bandwidth of $150 \mathrm{MHz}$ and with a $K=2{ }^{1} 2$ frequency samples.

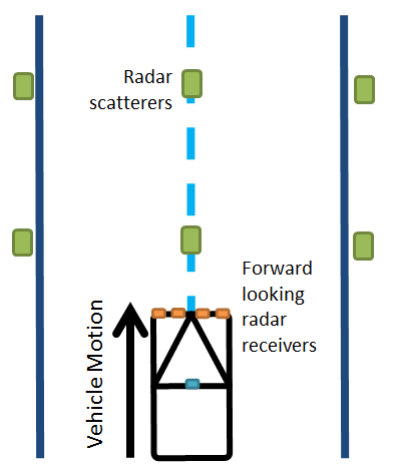

Fig. 6: The proposed configuration for the forward looking SAR scenario. The green squares are the radar reflectors, while the orange boxes are the SAR sensors. This figure is not presented to scale.

We test the first scenario using a stationary vehicle, with 16 receiver elements located across the front of the vehicle (hence $N_{p}=16$ ). Given that the wavelength of a $24 \mathrm{GHz}$ automotive radar is $1.25 \mathrm{~cm}$, it is entirely feasible to mount 16 (or even more) receive antennas across the front of a vehicle (though the RF receivers and processors may become problematic). In this scenario, the system is essentially operating in a multi-static radar mode, however we utilize the same image formation process as for SAR. The markers are spaced in locations at the center of the lane and at the edges edge of the lanes $( \pm 2 m)$ from the center, at spacing of $20 \mathrm{~m}$ apart. The results are displayed in Fig. 7, where the SAR image consists of both markers and aliasing effects.

The aliasing presents an issue as the peaks in the SAR image will be extracted as false-positive measurements. Essentially, the aliasing is caused by there being an insufficient number of angles at which the targets are viewed from, leading to ambiguities in their location. The aliasing can be overcome by randomizing the position of the radar receivers (or reflectors), introducing more receivers measurements (physically adding more receivers, or moving the receivers as a function of time).

As it is physically difficult to either add new receivers or randomize their location, the next scenario considers the image formation. Essentially, we set up the simulation in a similar way, with 16 receive elements over the front of the vehicle, but measured three times at 3 intervals of 10 meters moving towards the scatterers. The scenario and results are shown in Fig. 8, where $N_{p}=48$. The figure indicates that the aliasing has been almost completely eliminated during formation of the SAR image. This is essentially because the targets are now seen with a much larger angular diversity. While the results presented here are not comprehensive, they are sufficient to indicate the feasibility of a moving vehicle forming a SAR image to detect lane and carriageway markers to the front of the vehicle.

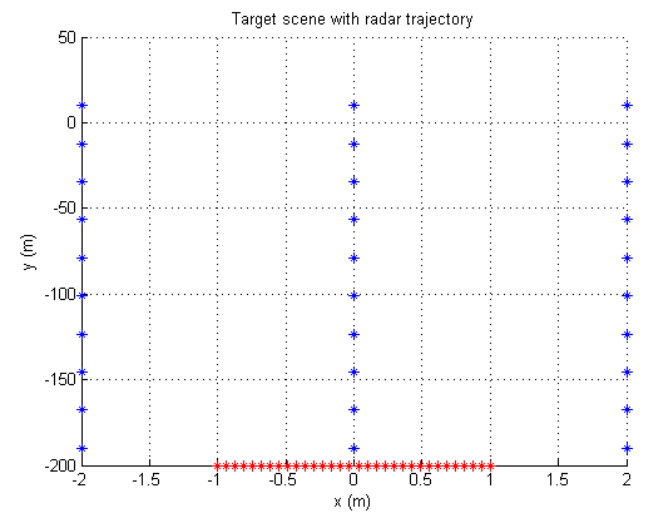

(a) Scene set up

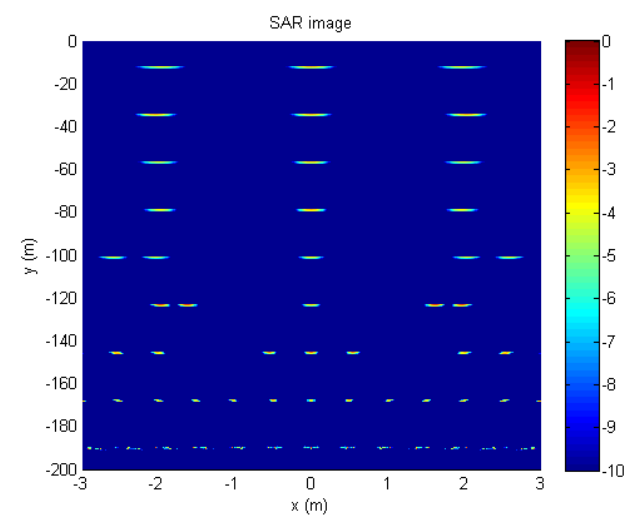

(b) SAR image

Fig. 7: A simulated scenario featuring a vehicle with a SAR observing lane markers in front of the vehicle. In this scenario, only a single layer of radar measurements are used simulating a stationary vehicle.

\section{Summary of Results}

In this section, we have used simulated scenarios to test the feasibility of using SAR for the detection of radar scatterers for lane and carriageway boundary detection. The simulated scenarios considered two cases of both a side and forward looking SAR forming an image of radar scatterers arranged similar to road markings. While neither the simulation or results are neither comprehensive they do prove the feasibility and act as a platform for further investigation.

Within automotive sensing scenarios, the scenes will be highly cluttered with objects which may or may not have 


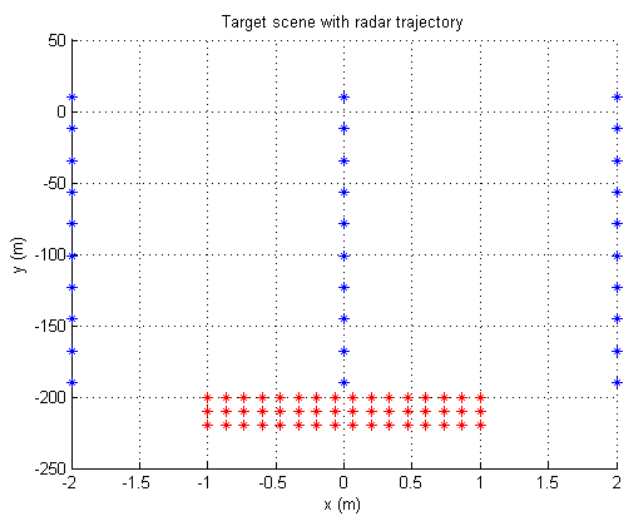

(a) Scene set up

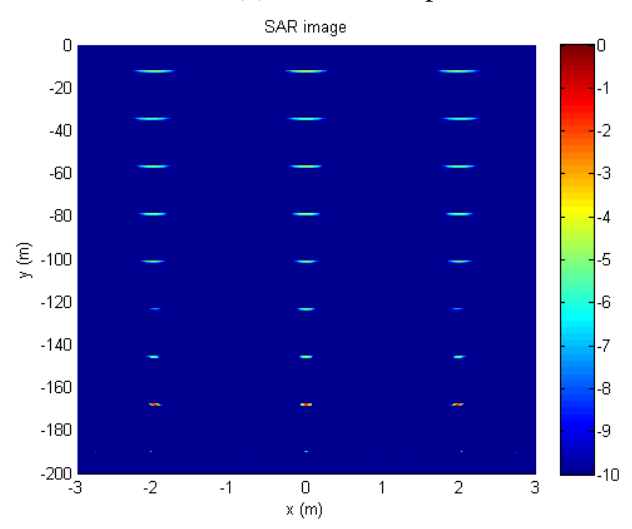

(b) SAR image

Fig. 8: A simulated scenario featuring a vehicle with a SAR observing lane markers in front of the vehicle. In this case, three layers of radar measurements are utilized to simulate a vehicle moving forward.

relevance to the driving application. While it is not investigated specifically within this paper, we can look to the results presented for the forward looking section to infer that an array of radar receivers which is also moving will provide a very high angular diversity of targets. Therefore, we expect that there will be sufficient resolution to fully resolve both the clutter and the radar reflectors.

\section{COnClusions}

In this paper we have presented preliminary work for using a Synthetic Aperture Radar to form a high resolution image of radar scatterer locations. We have shown using representative simulations that this technique is feasible for the detection of lane and carriageway markers under the assumption that the infrastructure is modified with embedded radar scatterers. This work has great promise as it reduces the reliance on traditional camera sensors whose performance degrades in conditions of poor visibility (e.g. low light conditions, poor weather etc.). In contrast, radar sensors do not suffer the same level of degradation and maintain performance during conditions of poor visibility.

This paper only indicates the feasibility of this technique.
Significant further work is clearly required and a number of practical obstacles (such as embedding retro-reflectors into road surfaces) remain to be overcome. Practical results are currently being generated in order to better appreciate the real world application of this technique. Additional work is also required in order to understand the effect of noise, clutter and the effect of other targets in the environment such as pedestrians, other vehicles and other infrastructure targets. It is envisaged that the detection of radar scatterers in lane and carriageway boundaries, in conjunction with Synthetic Aperture Radar could significantly aid advanced driver assistance systems such as lane departure warning systems which rely on their detection. Future work will consider the effect of noise and clutter and prove the feasibility of the technique using a real radar hardware.

\section{REFERENCES}

[1] Y. Sebsadji, J.-P. Tarel, P. Foucher, and P. Charbonnier, "Robust road marking extraction in urban environments using stereo images," in 2010 IEEE Intelligent Vehicles Symposium, pp. 394-400, IEEE, jun 2010.

[2] T. Veit, J.-P. Tarel, P. Nicolle, and P. Charbonnier, "Evaluation of Road Marking Feature Extraction," in 2008 11th International IEEE Conference on Intelligent Transportation Systems, pp. 174-181, IEEE, oct 2008.

[3] C. Kelber, "A lane departure warning system based on a linearparabolic lane model," in IEEE Intelligent Vehicles Symposium, 2004, pp. 891-895, IEEE.

[4] J. Huang, H. Liang, Z. Wang, Y. Song, and Y. Deng, "Lane marking detection based on adaptive threshold segmentation and road classification," in 2014 IEEE International Conference on Robotics and Biomimetics (ROBIO 2014), pp. 291-296, IEEE, dec 2014.

[5] J. McCall and M. Trivedi, "An integrated, robust approach to lane marking detection and lane tracking," in IEEE Intelligent Vehicles Symposium, 2004, pp. 533-537, IEEE.

[6] J. McCall and M. Trivedi, "Video-based lane estimation and tracking for driver assistance: survey, system, and evaluation," Intelligent Transportation Systems, ..., 2006.

[7] J. Gong, A. Wang, Y. Zhai, G. Xiong, P. Zhou, and H. Chen, "High Speed Lane Recognition under Complex Road Conditions," in 2008 IEEE Intelligent Vehicles Symposium, pp. 566-570, IEEE, jun 2008.

[8] F. Stein, "The challenge of putting vision algorithms into a car," in 2012 IEEE Computer Society Conference on Computer Vision and Pattern Recognition Workshops, pp. 89-94, IEEE, jun 2012.

[9] C. Wagner, J. Bock, M. Wojnowski, H. Jager, J. Platz, M. Treml, F. Dober, R. Lachner, J. Minichshofer, and L. Maurer, "A $77 \mathrm{GHz}$ automotive radar receiver in a wafer level package," in 2012 IEEE Radio Frequency Integrated Circuits Symposium, pp. 511-514, IEEE, jun 2012.

[10] Y. Takeda, T. Fujibayashi, W. Wang, and B. Floyd, "A 76- to 81-GHz transceiver chipset for long-range and short-range automotive radar," in 2014 IEEE MTT-S International Microwave Symposium (IMS2014), pp. 1-3, IEEE, jun 2014

[11] B.-H. Ku, O. Inac, M. Chang, and G. M. Rebeiz, "7585 GHz flip-chip phased array RFIC with simultaneous 8-transmit and 8-receive paths for automotive radar applications," in 2013 IEEE Radio Frequency Integrated Circuits Symposium (RFIC), pp. 371-374, IEEE, jun 2013.

[12] J. Hasch, E. Topak, R. Schnabel, T. Zwick, R. Weigel, and C. Waldschmidt, "Millimeter-Wave Technology for Automotive Radar Sensors in the $77 \mathrm{GHz}$ Frequency Band," IEEE Transactions on Microwave Theory and Techniques, vol. 60, pp. 845-860, mar 2012.

[13] J. Dickmann, J. Klappstein, M. Hahn, M. Muntzinger, N. Appenrodt, C. Brenk, and A. Sailer, "'Present research activities and future requirements on automotive radar from a car manufacturers point of view"," in 2015 IEEE MTT-S International Conference on Microwaves for Intelligent Mobility (ICMIM), pp. 1-4, IEEE, apr 2015.

[14] L. Nguyen, D. Wong, M. Ressler, F. Koenig, B. Stanton, G. Smith, J. Sichina, and K. Kappra, "<title $>$ Obstacle avoidance and concealed target detection using the Army Research Lab ultra-wideband synchronous impulse reconstruction (UWB SIRE) forward imaging 
radar $<$ title $>$," in Defense and Security Symposium (R. S. Harmon, J. T. Broach, and J. H. Holloway, Jr., eds.), pp. 65530H-65530H-8, International Society for Optics and Photonics, apr 2007.

[15] C. H. Jung, J.-H. Song, and H.-C. Lee, "Experimental X-Band Automotive SAR System for Land Observation Application," in SPACOMM 2015, The Seventh International Conference on Advances in Satellite and Space Communications, pp. 90-93, apr 2015.

[16] Y. Luo, H. Song, W. Robert, and S. Zheng, "High-resolution automobile FMCW SAR and signal processing," Journal of Electronics (China), vol. 30, pp. 561-566, dec 2013.

[17] "Intelligent mobility road to be built at cranfield university." Accessed: 2016-05-30.

[18] "Smart adaptive data aggregation (sada)." Accessed: 2016-05-30.

[19] F. Zhang, G. Hinz, D. Clarke, and A. Knoll, "Vehicle-Infrastructure Localization Based on the SME Filter," in 2015 IEEE 18th International Conference on Intelligent Transportation Systems, pp. 225-230, IEEE, sep 2015.

[20] F. Zhang, G. Hinz, D. Gulati, D. Clarke, and A. Knoll, "Cooperative vehicle-infrastructure localization based on the symmetric measurement equation filter," GeoInformatica, vol. 20, pp. 159-178, jan 2016.

[21] L. Gorham and L. Moore, "SAR image formation toolbox for MATLAB," SPIE Defense, ..., 2010.

[22] C. V. Jakowatz, Jr., D. E. Wahl, D. A. Yocky, B. K. Bray, W. J. Bow, Jr., and J. A. Richards, " $<$ title $>$ Comparison of algorithms for use in real-time spotlight-mode SAR image formation $<$ /title $>$," in Defense and Security (E. G. Zelnio and F. D. Garber, eds.), pp. 108116, International Society for Optics and Photonics, sep 2004. 